\title{
Preparation and Characterization of Super-toughened Poly(lactic acid)/cross-linked Polyurethane Blends via One-step Dynamic Vulcanization
}

\author{
Mengjie Sheng,,${ }^{1,3,4}$ Lida $\mathrm{Wu},{ }^{2}$ Xiaolong Li,,${ }^{1,3,4}$ Han Yan, ${ }^{2}$ Xiang $\mathrm{Lu},{ }^{1,3,4,{ }^{*}}$ Yang $\mathrm{Xu},{ }^{2} \mathrm{Yi} \mathrm{Li}^{2}{ }^{2}$ Yi Tong ${ }^{2, *}$ and Jinping $\mathrm{Qu}^{1,3,4,{ }^{*}}$
}

\begin{abstract}
In this study, a series of super-toughened poly(lactic acid) (PLA)/cross-linked polyurethane (CPU) blends were prepared via one-step dynamic vulcanization of PLA, polyethylene glycol (PEG) and isophorone diisocyanate trimer (IPDIT). FTIR and gel tests displayed that the CPU was successfully generated during the dynamic vulcanization reaction. The scanning electron microscopy (SEM) images of cryo-fractured and impacted surfaces showed that the PLA/CPU blends had no obvious phase separation, which indicated good compatibility between the PLA component and obtained CPU component. It also revealed the toughening mechanism dominated by shear yielding. The DSC results show the crystallinity of all the PLA/CPU blends was higher than neat PLA, indicating the CPU component can act as a crystallization nucleating agent for the PLA component. When the PEG content is $30 \%$, the impact strength and elongation at the break of PLA/CPU blends can be increased to 35 times and 42 times, respectively. All the results indicate the interfacial reaction and compatibilization between the PLA and CPU resulted in the formation of super-toughened PLA/CPU blended materials.
\end{abstract}

Keywords: Biodegradable; Super toughness; Poly(lactic acid); Cross-linked polyurethane; Dynamic vulcanization.

Received: 11 November 2021; Revised: 06 December 2021; Accepted: 07 December 2021.

Article type: Research article.

\section{Introduction}

Much attention to increasing environmental pollution was aroused to bio-based polymer materials regarded as substitutes for petroleum-based materials. ${ }^{[1-3]}$ Poly(lactic acid) (PLA), a kind of aliphatic polyester, has been widely used in many areas such as agriculture, textiles, and medical fields, owing to complete degradability, good biocompatibility, high mechanical strength, and easy to process. ${ }^{[4-7]}$ In particular,

\footnotetext{
${ }^{1}$ Hubei Engineering Research Center for Biomaterials and Medical Protective Materials, Huazhong University of Science \& Technology, Wuhan, 430074, China.

${ }^{2}$ COFCO(Jilin) Bio-Chemical Technology Co., Ltd., Changchun, 130000, China.

${ }^{3}$ Key Laboratory of Material Chemistry for Energy Conversion and Storage, Huazhong University of Science \& Technology, Ministry of Education, Wuhan, 430074, China.

${ }^{4}$ Hubei Key Laboratory of Material Chemistry and Service Failure, School of Chemistry and Chemical Engineering, Huazhong University of Science \& Technology, Wuhan, 430074, China.

*E-mail: luxiang@ hust.edu.cn (X. Lu),jpqu@ hust.edu.cn (J.P. Qu), Tongyi@cofco.com (Y. Tong)
}

several shortcomings such as the inherent brittleness of PLA limit its further application. ${ }^{[8-10]}$ Therefore, how to improve the toughness of PLA became a research hotspot.

Significant efforts have been devoted to toughening PLA so far, including chemical copolymerization, ${ }^{[11,12]}$ physical blending, ${ }^{[13]}$ plasticization, ${ }^{[14,15]}$ reactive blending, ${ }^{[11,16]}$ stereocomplex, ${ }^{[17,18]}$ etc. Blending modification is widely used and favored because of its simplicity, high efficiency, and easy to industrialize. Yet thermodynamically incompatibility among different polymers is responsible for the poor compatibility of polymer blends, ${ }^{[19,20]}$ which means that the desired effect is unavailable by simple physical blending. For example, due to the poor interfacial interaction, the elastomer toughening phase was usually detached from the PLA matrix during the impact of external force. ${ }^{[21]}$ The interfacial compatibilizers can significantly improve the interaction force between the blending components. ${ }^{[22]}$ In recent years, dynamic vulcanization technology based on reactive blending has become one of the most promising methods for preparing high-toughness PLA materials. because the interfacial compatibilizers can be generated via the in-situ reaction during the blending process. ${ }^{[23]}$ The rubber phase is not only 
selectively vulcanized but also sheared into micro/nanoscale rubber particles, which can act as stress concentration points to achieve high-efficiency toughening of the PLA. ${ }^{[24]}$ Liu et al. prepared a series of super-toughened polymers via dynamic vulcanization with PLA, ethylene/n-butyl acrylate/glycidyl methacrylate copolymer (EBA-GMA), and zinc ionomer of ethylene/methacrylic acid copolymer (EMAA-Zn). They found that the EBA-GMA rubber phase can be cross-linked by EMAA-Zn, and meanwhile be grafted into the PLA at the interface between PLA and EBA-GMA to effectively improve the interface interaction and notched impact strength of PLA/EBA-GMA blends. ${ }^{[25]}$ Jiang et al. dynamic vulcanized PLA and epoxidized natural rubber (ENR) under the induction of peroxide, and obtained a series of super-toughened PLA/ENR blends with a co-continuous structure. ${ }^{[26]}$ All in all, dynamic vulcanization based on reactive blending has unique advantages in the toughening of PLA.

In addition, polyurethane (PU) elastomer is an ideal material for medical devices because of its flexibility and biocompatibility. ${ }^{[27-30]}$ However, due to the poor interface interaction between PU and PLA, ${ }^{[31,32]}$ the toughening effect on the PLA matrix is generally unacceptable. To improve the interface interaction between PU and PLA, and obtain supertoughened PLA/PU blends, the dynamic vulcanization based on PLA/PU blends system is widely reported in the literature. ${ }^{[33-35]}$ Polyurethane elastomer prepolymer (PUEP) is a linear or cross-linked intermediate product of $\mathrm{PU}$, which is terminated by reactive isocyanate groups $(-\mathrm{NCO})$. Under appropriate reaction conditions, PUEP can be vulcanized to a rubber phase with a three-dimensional net-like structure. Lu et al. prepared a series of PLA/PU blends via the dynamic vulcanization of PLA and PUEP systems. During the dynamic vulcanization process, a small amount of PLA-g-PU copolymer is also formed, and the interface interaction between PLA and PU is significantly improved under its action. ${ }^{[36]}$ In addition, Shakouri et al. also reported the toughness of PLA/PUEP/cellulose nanocrystal composites via dynamic vulcanization. ${ }^{[37]}$ However, what the problems remain are that a large technical difficulty exists in the synthesis process of specific PUEP ${ }^{[38]}$ and molecular structure design also suffers some limitations. Therefore, it is of great significance to develop an efficient and flexible dynamic vulcanization process to toughen PLA.

In this work, polyethylene glycol (PEG) and isophorone diisocyanate trimer (IPDIT) were employed as soft segments and hard segments respectively. Through the one-step dynamic vulcanization process of the PLA/PEG/IPDIT system, the cross-linked PU and a small amount of PLA-g-CPU were in-situ synthesized, and a series of super-toughened PLA/CPU blends were obtained. Simultaneously, the interface interaction between PLA and CPU was significantly improved by in situ formed PLA-g-CPU. The influence of PEG molecular weight and content, and the ratio of $-\mathrm{NCO} /-\mathrm{OH}$ on the micro-phase morphology, mechanical properties, and thermal properties of the PLA/CPU blends were discussed. To the best of our knowledge, a similar reactive compatibilizing and toughening route for super-toughened PLA/CPU blends through dynamic vulcanization has not yet been reported. The research results provide important theoretical value for the preparation of high-performance PLA materials.

\section{Materials and methods \\ 2.1 Materials}

PLA (2003D) was purchased from NatureWorks LLC (Minnetonka, MN). PEG1000, PEG4000, PEG10000, and PEG20000, were obtained from Shanghai Macklin Biochemical (China) Co., Ltd. Isophorone diisocyanate trimer (IPDIT) was obtained from Covestro Co., Ltd. Dichloromethane and Dibutyltin dilaurate was obtained from Shanghai Macklin Biochemical (China) Co., Ltd.

\subsection{Sample preparation}

Before processing, PLA and PEG were dried at $80{ }^{\circ} \mathrm{C}$ under a vacuum for $8 \mathrm{~h}$. PLA/CPU blends with various PEG loading, PEG molecular weight, and the molar ratio of isocyanate groups from IPDIT to hydroxyl groups from PEG were meltcompounded by using a HAAKE torque rheometer with a chamber volume of $55 \mathrm{~cm}^{3}$ and roller blades at $180{ }^{\circ} \mathrm{C}$. The rotor speed was kept at $60 \mathrm{rpm}$ for $15 \mathrm{~min}$. PEG and catalyst were added after PLA was completely melted for about $3 \mathrm{~min}$. Then, IPDIT was added after PLA and PEG were blended uniformly, and the blending was continued for another $5 \mathrm{~min}$. All blends were immediately cooled and cut into pellets. These pellets were dried at $80^{\circ} \mathrm{C}$ for more than $4 \mathrm{~h}$. After being ovendried, the pellets were injection-molded into specimens. The temperature profile of the injection barrels was $170{ }^{\circ} \mathrm{C} / 165^{\circ} \mathrm{C} / 160^{\circ} \mathrm{C}$ from the first heating zone to the nozzle. The injection pressure was set at $20 \mathrm{MPa}$. All test specimens were conditioned for 7 days at $25{ }^{\circ} \mathrm{C}$ and $50 \%$ relative humidity prior to testing and characterization. The detailed formulations of the PLA/CPU blends were listed in Table 1.

Table 1. The formulations of PLA/CPU blends.

\begin{tabular}{cccc}
\hline Code & PLA (wt \%) & PEG (wt \%) & $\begin{array}{c}\text { IPDIT } \\
\text { (wt \%) }\end{array}$ \\
\hline Neat PLA & 100 & 0 & 0.0 \\
$1 \mathrm{~K} / 10 \% / 1.1$ & 90 & 10 & 7.9 \\
$1 \mathrm{~K} / 20 \% / 1.1$ & 80 & 20 & 15.8 \\
$1 \mathrm{~K} / 30 \% / 0.8$ & 70 & 30 & 17.2 \\
$1 \mathrm{~K} / 30 \% / 1.1$ & 70 & 30 & 23.7 \\
$1 \mathrm{~K} / 30 \% / 1.3$ & 70 & 30 & 28.1 \\
$4 \mathrm{~K} / 30 \% / 1.1$ & 70 & 30 & 5.8 \\
$1 \mathrm{~W} / 30 \% / 1.1$ & 70 & 30 & 2.3 \\
$2 \mathrm{~W} / 30 \% / 1.1$ & 70 & 30 & 1.2 \\
\hline
\end{tabular}

\subsection{Characterization}

\subsubsection{Gel content measurement}

Samples with a predetermined weight $\left(M_{i} \approx 1 \mathrm{~g}\right)$ were cut into very small pellets. The extraction was performed by using a Soxhlet extractor for 3 days with an excess volume of boiling 
dichloromethane. The fraction of the sample that did not dissolve in dichloromethane but just swelled was then weighed $\left(M_{f}\right)$ after complete drying under vacuum at room temperature. The insoluble fraction must consist of the vulcanized PU and possibly PLA chains, which reacted with PU. The Gel fraction was calculated according to Eq. (1). ${ }^{[39]}$

$$
\text { Gel content }(\%)=\frac{M_{f}}{M_{i}} \times 100 \%
$$

\subsubsection{Fourier transform infrared analysis (FTIR)}

FTIR spectra of the PLA/CPU blends and insoluble sample fractions were recorded at room temperature using Fouriertransform infrared spectroscopy (FTIR, Nicolet iS5, USA). And the FTIR was used to analyze the chemical structures in the wavenumber range of $500-4000 \mathrm{~cm}^{-1}$.

\subsubsection{Scanning electron microscopy (SEM)}

The morphologies of blends were examined by scanning electron microscopy (FE-SEM, Hitachi SU8010) at an accelerating voltage of $5 \mathrm{kV}$. The injection-molded bars were cryo-fractured in liquid nitrogen and then sputter-coated with a thin layer of gold prior to examination. The fractured surfaces after the impact test were also observed by the same SEM apparatus.

\subsubsection{Differential Scanning Calorimetry (DSC)}

A differential scanning calorimeter (DSC, TA 25, USA) was used to examine the crystallinity degree. The samples, with weight in the range of 7-9 $\mathrm{mg}$, were melted at $200{ }^{\circ} \mathrm{C}$ for $5 \mathrm{~min}$ and then cooled to $0{ }^{\circ} \mathrm{C}$ at a rate of $10{ }^{\circ} \mathrm{C} / \mathrm{min}$. After cooling, the polymers were subsequently heated to $200^{\circ} \mathrm{C}$ at $10^{\circ} \mathrm{C} / \mathrm{min}$. During the DSC runs, a nitrogen flow at a rate of $50 \mathrm{~mL} / \mathrm{min}$ was constantly applied. The crystallinity degree $X_{c}(\%)$ of the PLA component in the different blends were calculated using the following formula:

$$
X_{c}(\%)=\frac{\Delta H_{m}-\Delta H_{c c}}{\Delta H_{m}{ }^{\circ} \times W_{f}} \times 100 \%
$$

where $\Delta H_{m}$ and $\Delta H_{c c}$ are the measured enthalpies of melting and cold crystallization for the PLA phase in the blends, $\Delta H_{m}^{\circ}$ is the melting enthalpy of $100 \%$ crystalline PLA $(93.7 \mathrm{~J} / \mathrm{g})$, and $W_{f}$ is the weight fraction of PLA in the blend.

\subsubsection{Thermogravimetric Analysis (TGA)}

Thermogravimetric analysis (TGA, TA 55, USA) was performed under a nitrogen atmosphere at heating rates of $10{ }^{\circ} \mathrm{C} /$ min ranging from $30^{\circ} \mathrm{C}$ to $700{ }^{\circ} \mathrm{C}$.

\subsubsection{Mechanical properties measurements}

The tensile strength of samples was tested at room temperature according to the ISO 527-5:1997 standards for modified material splines. The tensile speed was $50 \mathrm{~mm} / \mathrm{min}$. The impact strengths of samples were tested at room temperature according to the ISO 180:2019 standard with a notch, the pendulum impact angle was $-150^{\circ}$, and the average value of five tests for each performance test result was taken as the final value.

\section{Result and discussion \\ 3.1 Reaction mechanism}

The molecular structure of IPDIT Contains three isocyanates, which are very easy to react with $-\mathrm{OH}$ from PEG or PLA. ${ }^{[40,41]}$ Therefore, when dynamic vulcanization between PLA, PEG, and IPDIT occurred, not only CPU was generated in-situ via the reaction between PEG and IPDIT, but also the PLA-g-PEG copolymer was generated as interface compatibilizer via the reaction between PEG, IPDIT, and PLA. ${ }^{[42]}$

Figure 1a shows the torque curve with time during the dynamic vulcanization of the PLA/CPU blends. It could be clearly seen that the whole process is divided into three stages. The first stage was the melting process of PLA pellets in the torque rheometer. As time went by, the curve first increased and then decreased. The torque value decreased rapidly with the addition of PEG in the second stage, which was attributed to the lubrication and plasticization of PEG. ${ }^{[43]}$ The third stage was the in-situ reaction process of IPDIT, PEG, and PLA. When IPDIT was added, the torque value dropped slightly. However, with the vulcanization process, the torque value increased gradually until remaining constant, suggesting the end of the vulcanization.

To confirm the formation of polyurethane (PU) during dynamic vulcanization, the FTIR spectra of $1 \mathrm{~K} / 30 \% / 1.1$ and its gel fraction were recorded and compared with neat PLA. From Fig. 1b, compared with neat PLA, a new absorption peak at $1540 \mathrm{~cm}^{-1}$ in the blends was the characteristic absorption peak of urethane, which sufficiently evidenced that polyurethane was successfully formed during blending. Accordingly, the isocyanate group peak in $2270 \mathrm{~cm}^{-1}$ could not be detected, which means the complete consumption of IPDIT. ${ }^{[4]}$ The N-H absorption peak at $3325 \mathrm{~cm}^{-1}$ and neat PLA carbonyl absorption peak at $1758 \mathrm{~cm}^{-1}$ could be seen in the FTIR spectra of Gel-1K/30\%/1.1, which indicated that the CPU was successfully generated during the dynamic vulcanization. Moreover, to a certain degree, the reaction between PLA and IPDIT achieved interface compatibilization. To verify the CPU generated in the blends, the cross-linked part of the PLA/CPU blends was extracted with dichloromethane, and the results are shown in Fig. 1c. Due to neat PLA without a cross-linked structure, the mass fraction gel was 0 . For PLA/CPU blends, the gel content increased with the increase of PEG content and - NCO/-OH. Remarkably, an increase in the molecular weight of PEG brought a decrease in gel content, since the decreased feed content of IPDIT will reduce the possibility of the reaction between PEG and IPDIT. We also found that the gel content of all blends is lower than the theoretical value when the PEG dosage is $30 \%$, indicating that only part of the PEG cloud efficiently participates in CPU synthesis.

The main components of gel may be cross-linked PLA and CPU, so TGA was performed to determine the content of the CPU. Figs. 1d-f show the TGA curves of insoluble fraction 

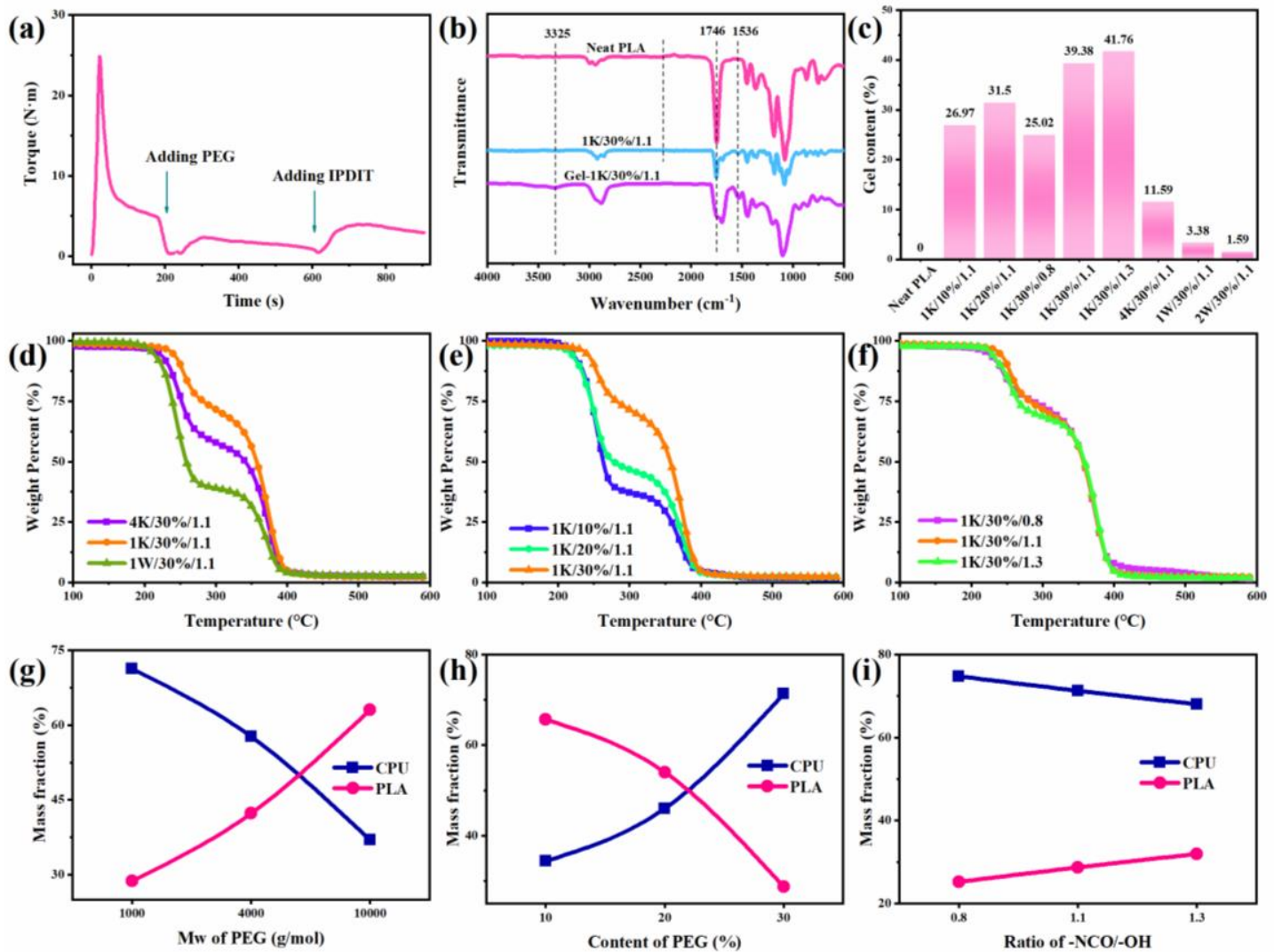

Fig. 1 (a) Torque versus time curves during the dynamic vulcanization; (b) FTIR spectra of the PLA/CPU blend and gel; (c) Gel content of the PLA/CPU blends; (d) - (f) TGA curves of gel; (g) - (i) Individual gel fraction of the PLA and CPU phases in gel.

with different PEG molecular weights, PEG content, and $\mathrm{NCO} /-\mathrm{OH}$ ratio. Two-stage weight loss can be observed in all the curves. The first stage and the second stage belong to PLA and CPU weightless, respectively. ${ }^{[43]}$ The mass fractions of cross-linked PLA and CPU calculated according to TGA curves are shown in Figs. 1g-i. As we all know, higher molecular weight PEG usually lower activity and contains fewer terminal hydroxyl groups under the same mass, which leads to a decrease in the CPU content of the blends. It is interesting to find that PLA content is higher than CPU when PEG contents are $10 \%$ and $20 \%$ in Fig. $1 \mathrm{~h}$, indicating that the less PEG content, the higher the reaction probability between IPDIT and PLA.

The performance of the polymer largely depends on phase morphology, and SEM can be used to observe the apparent morphology of the PLA/CPU blends. As shown in Fig. 2, neat PLA exhibited a smooth and clean surface. On the other hand, all the PLA/CPU blends, without "sea-island" structural and obvious phase separation, showed good compatibility. As the PEG content increased, the surface of the blends began to become rough, but no obvious phase separation was observed. The influence of the molecular weight of PEG on morphology is that the higher molecular weight of PEG led to worse interfacial adhesion. However, thanks to a large number of ether bonds and terminal hydroxyl groups of the PEG main chain, good compatibility still was a distinct feature in all PLA/CPU blends.

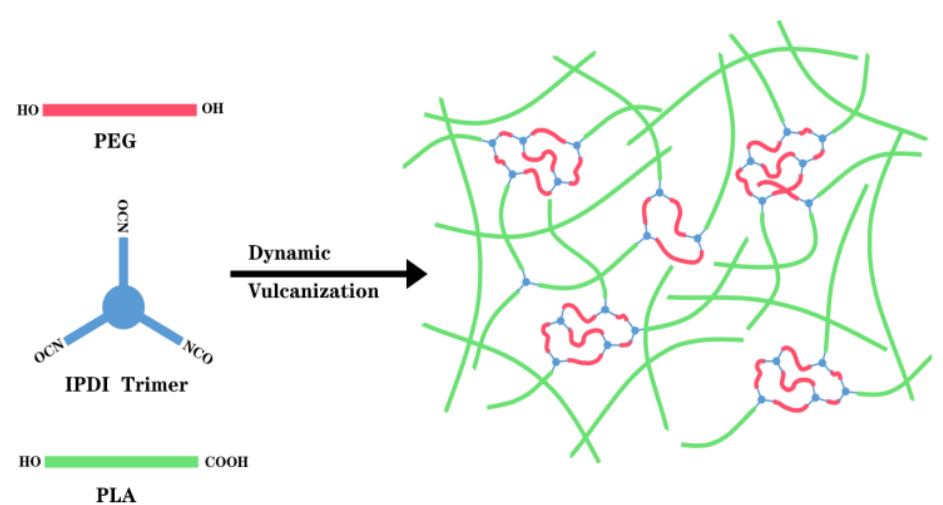

PLA

Scheme 1. Reaction mechanism of the PLA/CPU blends. 

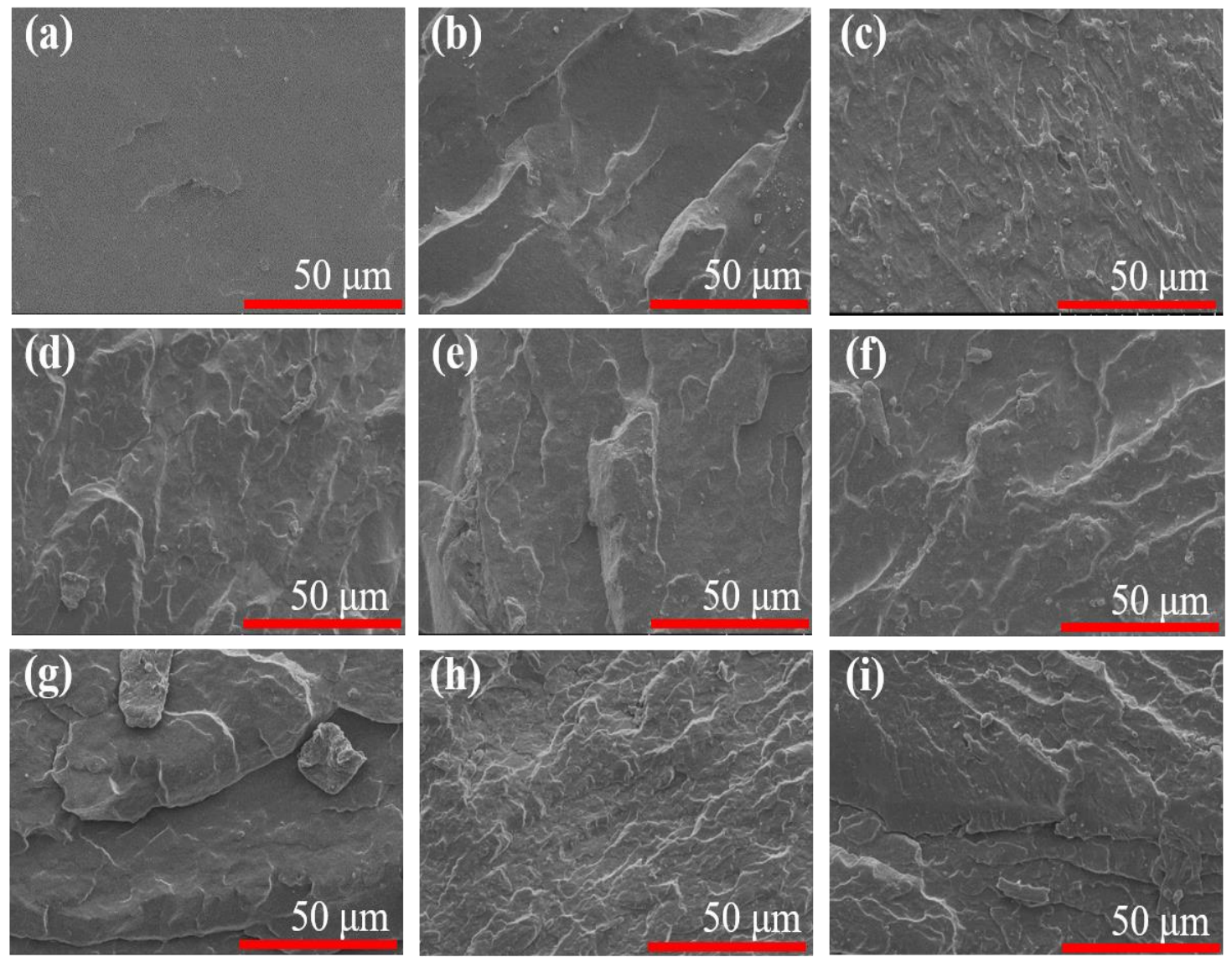

Fig. 2 SEM images of cryo-fractured surfaces for (a) Neat PLA, (b) $1 \mathrm{~K} / 10 \% / 1.1$, (c) $1 \mathrm{~K} / 20 \% / 1.1$, (d) $1 \mathrm{~K} / 30 \% / 0.8$, (e) $1 \mathrm{~K} / 30 \% / 1.1$, (f) $1 \mathrm{~K} / 30 \% / 1.3$, (g) $4 \mathrm{~K} / 30 \% / 1.1$, (h) $1 \mathrm{~W} / 30 \% / 1.1$, (i) $2 \mathrm{~W} / 30 \% / 1.1$.

The principle of the dynamic vulcanization process to prepare the PLA/CPU blends is shown in Scheme 1. The $\mathrm{NCO}$ is the characteristic functional group of IPDIT, easily reacting with the $-\mathrm{OH}$ groups in $\mathrm{PEG}$ to form polyurethane. Concurrently IPDIT also acts as a chemical crosslinking point to form a cross-linked polyurethane dispersed in the PLA matrix. Moreover, PLA-g-CPU generated during dynamic vulcanization, can achieve in-situ compatibilization by bridging the two phases of PLA and CPU, effectively enhancing the interface force.

\subsection{The influence of PEG molecular weight on the properties of blends}

The molecular weight of PEG is highly related to the length of the soft segment in the CPU, which directly affects the flexibility of the CPU. ${ }^{[45]}$ To explore the effect of different flexible CPUs on the toughening effect of PLA, we selected PEG with different molecular weights as the raw materials to study the mechanical properties of the blends. As shown in Fig. $3 \mathrm{a}$, the stress-strain curve of neat PLA showed typical brittle behavior clearly, with tensile strength and elongation of 60 $\mathrm{MPa}$ and $11 \%$ respectively. With shifting the molecular weight of PEG from 1000 to 20000, the elongations of those blends were $246 \%, 234 \%, 396 \%$, and $459 \%$ respectively. However, the tensile strength of the blends decreased significantly as the molecular weight of PEG increased. The impact strength is considered a measure of the material's toughness. ${ }^{[46]}$ The results of neat PLA and the PLA/CPU blends are shown in Fig. $3 \mathrm{~b}$. The impact strength of neat PLA is only $2.31 \mathrm{~kJ} / \mathrm{m}^{2}$. After the introduction of the CPU, the impact strength of the blends first decreased and then increased with the molecular weight of PEG. The test results show that different PEG molecular weights can significantly improve the impact strength of the PLA/CPU blends. However, there is no obvious correlation between toughness and molecular weight. To further explore the effect of molecular weight on blends, the crystallinity of blends was discussed next.

PLA is a kind of typical semi-crystalline polymer, whose crystallization process and crystallinity have a great influence on mechanical properties. Therefore, the crystallization behavior of the PLA/CPU blends was analyzed by DSC exhibited in Fig. 4, and thermal parameters are listed in Table S1. From Fig. $4 \mathrm{~b}$ of a heating process, PEG melting peaks appeared in the range of $40{ }^{\circ} \mathrm{C}-60{ }^{\circ} \mathrm{C}$ in the samples of 4 $\mathrm{K} / 30 \% / 1.1 /, 1 \mathrm{~W} / 30 \% / 1.1$, and $2 \mathrm{~W} / 30 \% / 1.1$. Fig. $4 a$ shows the cooling process of the blends. It is obvious that the PLA and PEG crystallization peaks appeared in $4 \mathrm{~K} / 30 \% / 1.1$, $1 \mathrm{~W} / 30 \% / 1.1$ and $2 \mathrm{~W} / 30 \% / 1.1$. This phenomenon may be caused by two reasons: 1 . The blend contains more unreacted PEG, which can self-crystallize and promote PLA 

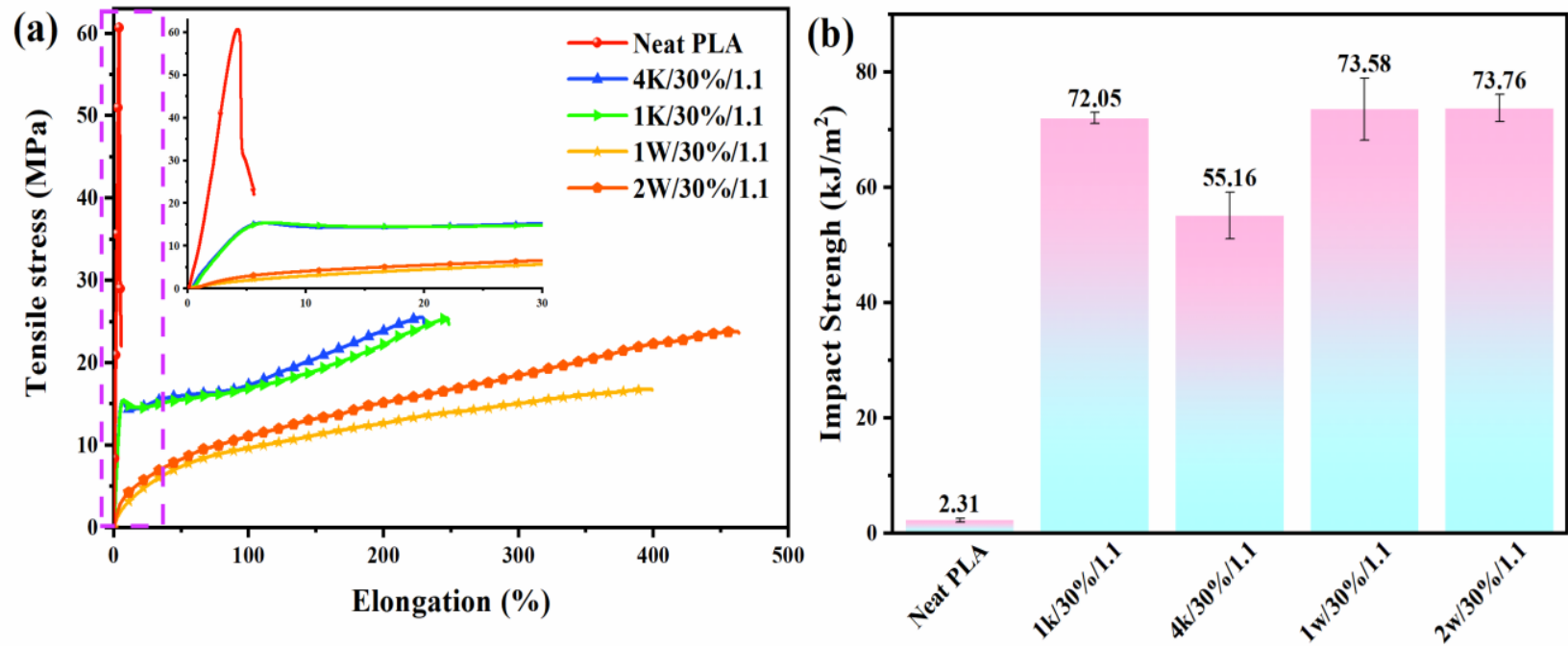

Fig. 3 (a) Tensile stress-strain curves of the blends with different molecular weights, (b) Impact strength of the blends with different molecular weights.

crystallization; 2. Even though PEG reacted with IPDIT, the middle segment of the chain of PEG was long enough to crystallize itself and improve the migration ability of PLA. When the molecular weights of PEG were 4000, 10000, and 20000 , the crystallinity was $27.4 \%, 45.21 \%$, and $49.87 \%$, respectively. Compared with the crystallinity of neat PLA $(5.10 \%)$, the crystallinity of the blends has significantly improved, which is consistent with the change in impact strength results. However, $1 \mathrm{~K} / 30 \% / 1.1$ with a crystallinity of $7.95 \%$ still had ultra-high impact strength, which was attributed to the fact that the high content of the CPU can effectively absorb external energy. ${ }^{[47]}$

\subsection{The influence of PEG content on the properties of blends}

To study the effect of CPU content on the properties of the PLA/CPU blends, the molecular weight of PEG and the ratio of $-\mathrm{NCO} /-\mathrm{OH}$ were fixed, and then the content of PEG in the system was changed from $10 \%$ to $30 \%$. Fig. 5a shows the stress-strain curves of the blends. With only $10 \%$ PEG added, the elongation exceeded $150 \%$, and the tensile strength was

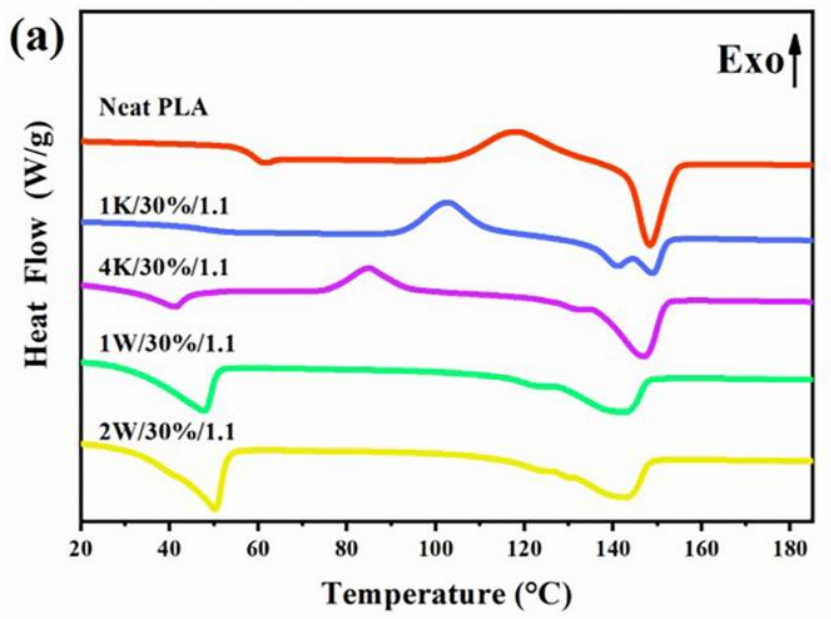

only reduced by $16 \%$. With the further increase of PEG content, the elongation at break first decreased and then increased, while the tensile strength had been declining. The impact strength results are shown in Fig. 5b. No matter whether the PEG content is $10 \%$ or $20 \%$, the impact strength is below $5 \mathrm{~kJ} / \mathrm{m}^{2}$. After $30 \%$ PEG was added, however, the impact strength was up to $72.05 \mathrm{~kJ} / \mathrm{m}^{2}$, which suggested that higher content of CPU would bring better toughness.

The DSC heating curves of the PLA/CPU blends with different CPU content are exhibited in Fig. 6b. The cold crystallization temperature of all blends had declined, indicating that the existence of the CPU was beneficial to the crystallization of PLA. In Fig. 6a, contrary to the previous study, there is no crystallization peak of PEG and PLA in the cooling process, demonstrating more reaction in the system. It can also be found that more CPU content could bring higher crystallinity from Table S2. Although the changing trend of crystallinity was consistent with the impact strength, there was no significant increase in crystallinity compared to neat PLA. Therefore, the reason why the impact strength varied with PEG content was owing to the increase in CPU content.

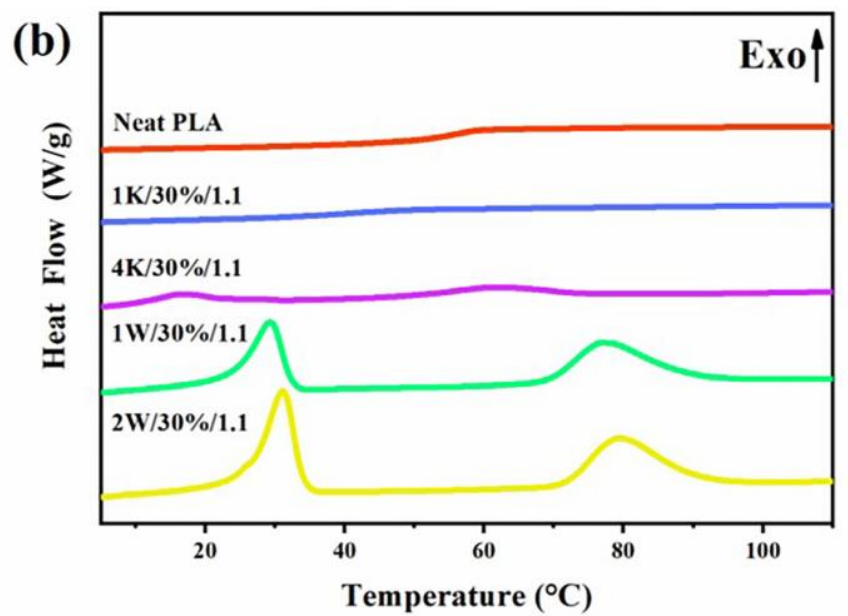

Fig. 4 The DSC heating (a) and cooling (b) curves of neat PLA and the blends with different molecular weights. 

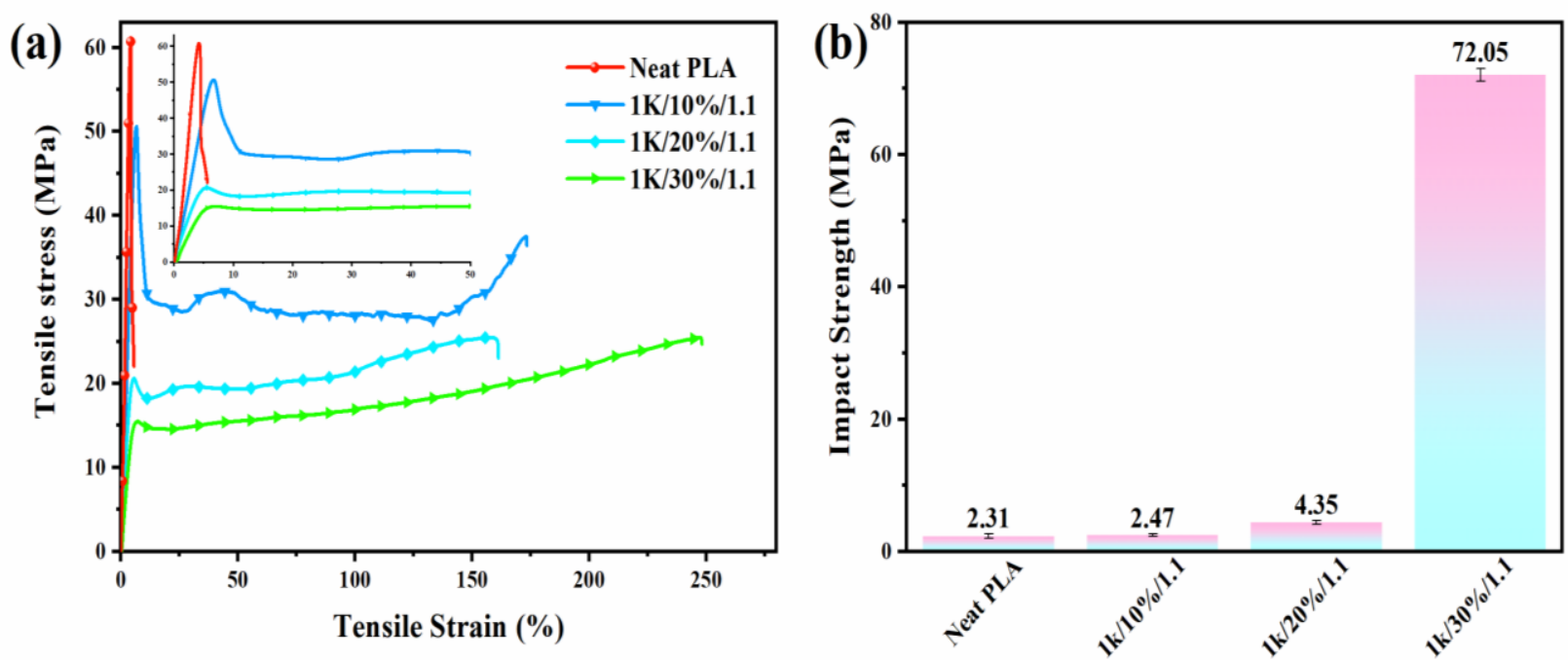

Fig. 5 (a) Tensile stress-strain curves of the blends with different PEG content, (b) Impact strength of the blends with different PEG content.

\subsection{The influence of $\mathrm{NCO} /-\mathrm{OH}$ on the toughening effect}

As we all know, the degree of cross-linking has a significant impact on the properties of the obtained materials. ${ }^{[48]}$ In this study, we adjusted the ratio of $-\mathrm{NCO} / \mathrm{-OH}$ to study how the degree of cross-linking affects the properties. Fig. 7a displays that when $-\mathrm{NCO} / \mathrm{-OH}$ was $0.8,1.1$, and 1.3 , the elongations at break were $285 \%, 246 \%$, and $334 \%$, respectively, which were 26 times, 22 times, and 30 times higher than neat PLA. Obviously, as a result of the substantial increase in elongation, the tensile strength exhibited an opposite trend. The results of the impact strength are shown in Fig. 7b. Overall, the impact strength increased first and then decreased. Interestingly, the impact strength was only $13.97 \mathrm{~kJ} / \mathrm{m}^{2}$ with fixing the ratio of $-\mathrm{NCO} /-\mathrm{OH}$ at 0.8 , which was much lower than those of 1.1 and 1.3. The excellent toughening effect of $1 \mathrm{~K} / 30 \% / 1.1$ and $1 \mathrm{~K} / 30 \% / 1.3$ was closely related to the degree of cross-linking. According to the gel results, as the $-\mathrm{NCO} / \mathrm{-OH}$ ratio increased, the gel mass fractions were $25.02 \%, 39.38 \%$, and $41.76 \%$, respectively. In other words, it is because of lower CPU content that $1 \mathrm{~K} / 30 \% / 0.8$ exhibited poor results of the impact strength.

The DSC heating curves of the PLA/CPU blends with

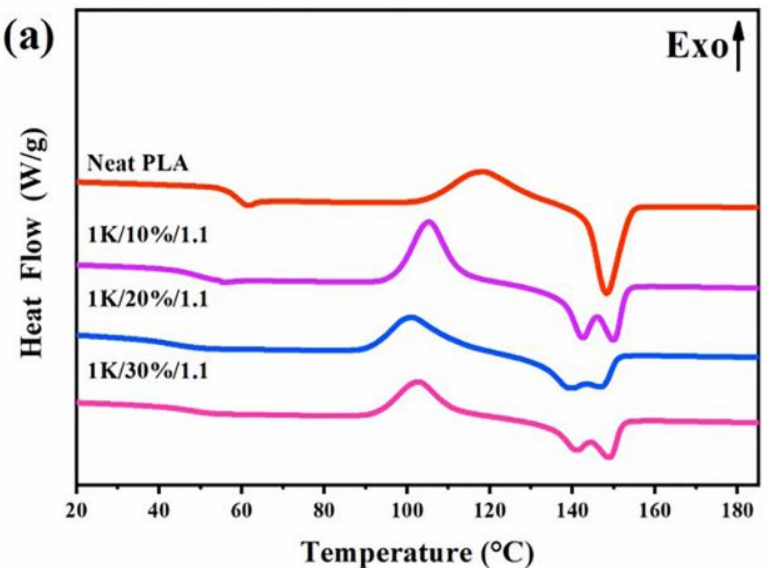

different crosslinking degrees are shown in Fig. 8b. According to the thermal parameters in Table S3, it was clearly observed that the higher degree of cross-linking caused lower crystallinity. When $-\mathrm{NCO} /-\mathrm{OH}$ was 0.8 , excessive PEG not only promoted PLA chain migration ability but also served as heterogeneous nucleation points to crystallize PLA. There was no marked change in the CPU content with varying - NCO/$\mathrm{OH}$ from 1.1 to 1.3. However, the higher degree of crosslinking would hinder the movement of the PLA chain, leading to a lower crystallinity. Yet the crystallinity of those blends was almost identical, which indicated that the crystallinity was not an important factor in toughness here.

\subsection{Toughening mechanism}

In previous research, the results of mechanical properties demonstrated a great improvement in toughness. For what concerned the PLA/CPU blends, the toughening mechanism about how the CPU affects the blends was still an issue. SEM was used to photograph the three areas of the impact fractured surfaces of the samples, and respectively marked as $1 / 2 / 3$, which is shown in Scheme 2. The impact sections are shown in Fig. 9. The three sections of neat PLA are very smooth,

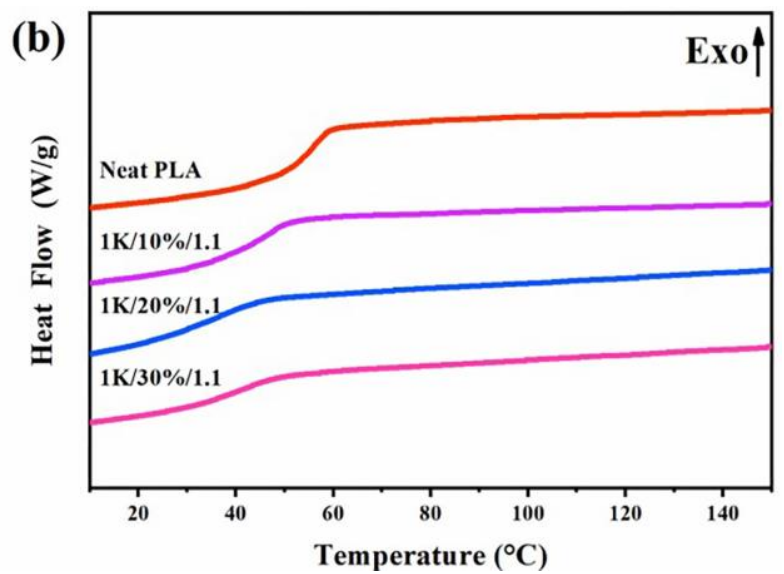

Fig. 6 The DSC heating (a) and cooling (b) curves of neat PLA and the blends with different PEG content. 

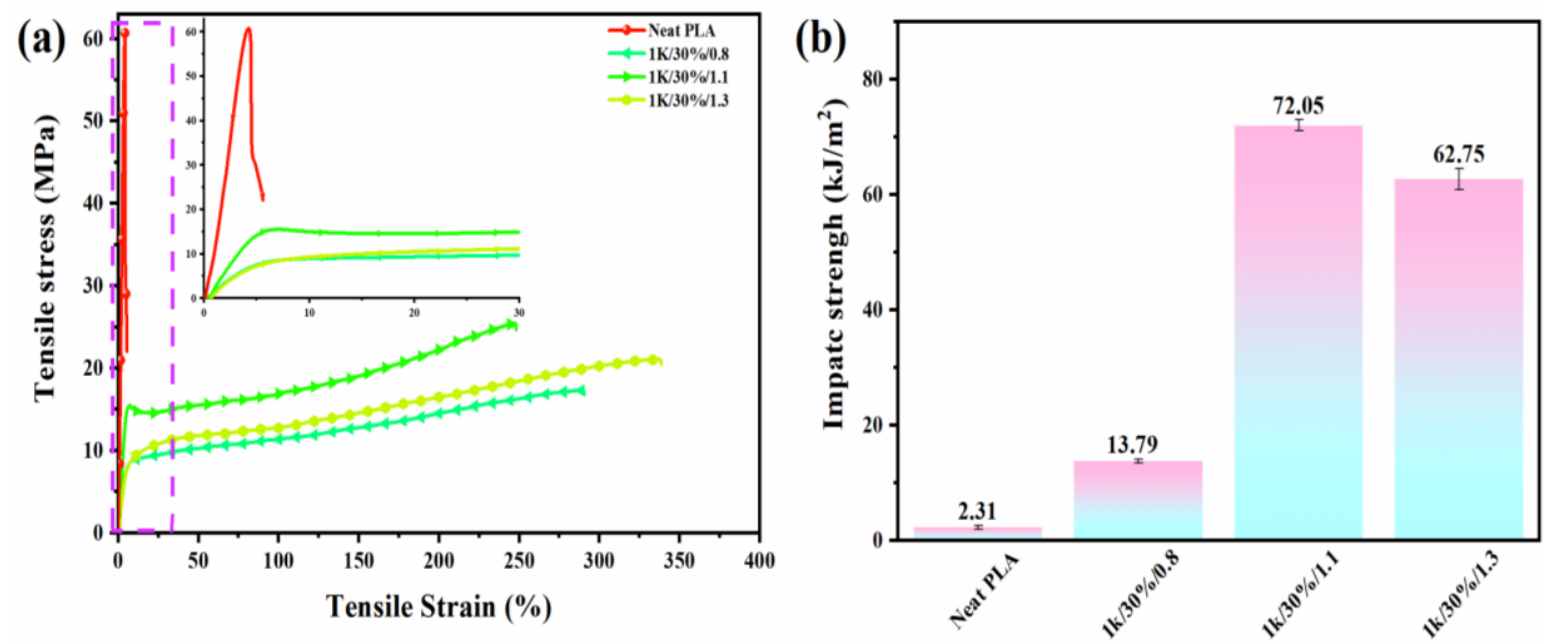

Fig. 7 (a) Tensile stress-strain curves of the blends with different -NCO/-OH, (b) Impact strength of the blends with different -NCO/$\mathrm{OH}$.

which is characteristic of brittle fracture. On the contrary, the surfaces of the PLA/CPU blends are relatively rough, obviously indicating the characteristic of ductile fracture. Crazing and shear yielding are the two main mechanisms of rubber toughening polymers. ${ }^{[49,50]} \mathrm{CPU}$ formed by PEG as the

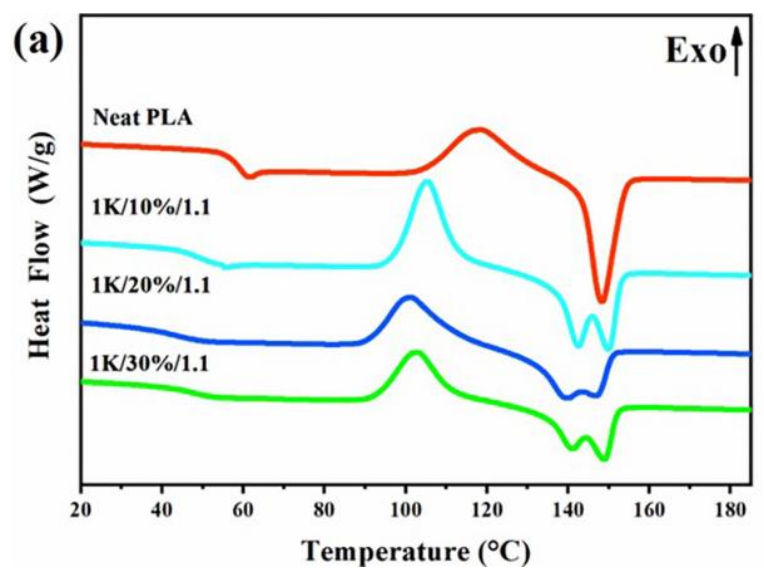

soft segment and IPDIT as the hard segment was dispersed in the PLA matrix as an elastic polymer. CPU can act as a stress concentration point in the PLA matrix. When impacted, the CPU not only induced a large number of shear zones and crazing and absorb a large amount of energy, but also

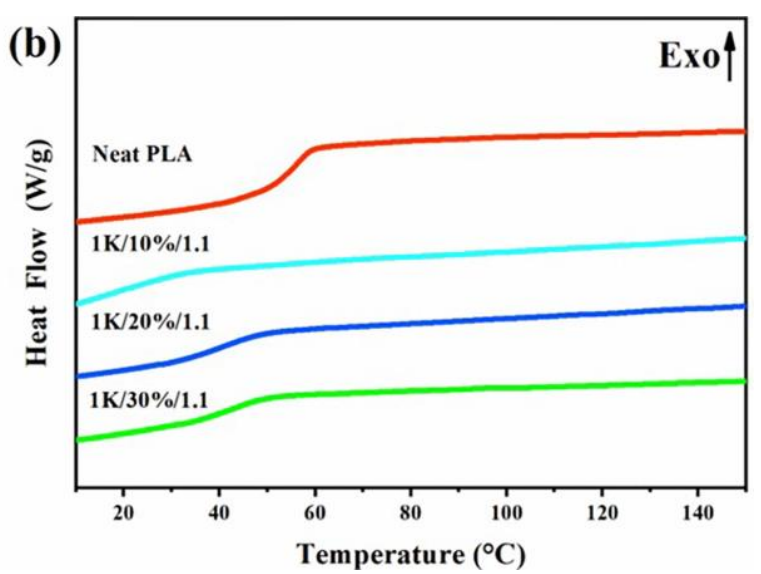

Fig. 8 The DSC heating (a) and cooling (b) curves of neat PLA and the blends with different -NCO/-OH.

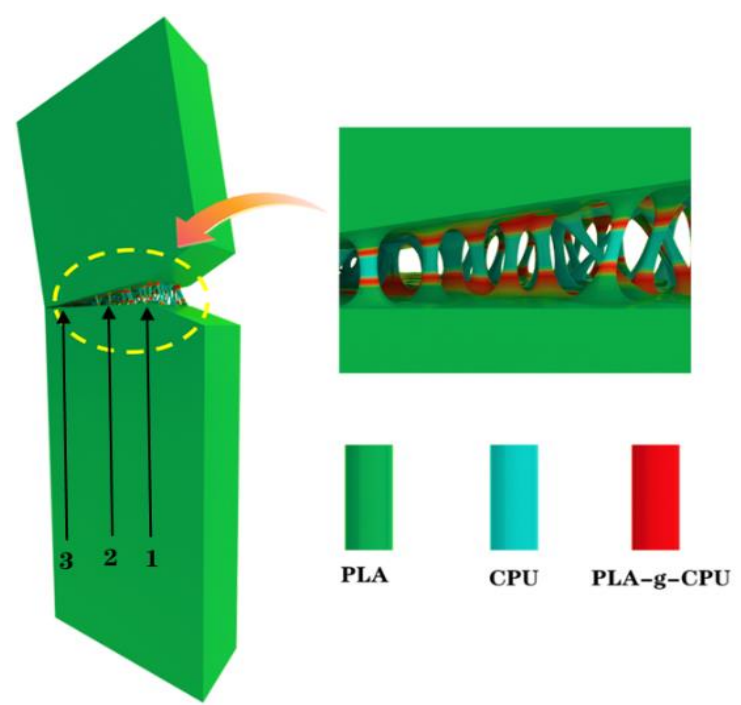

Scheme 2. Schematic diagram of fracture mechanism. 

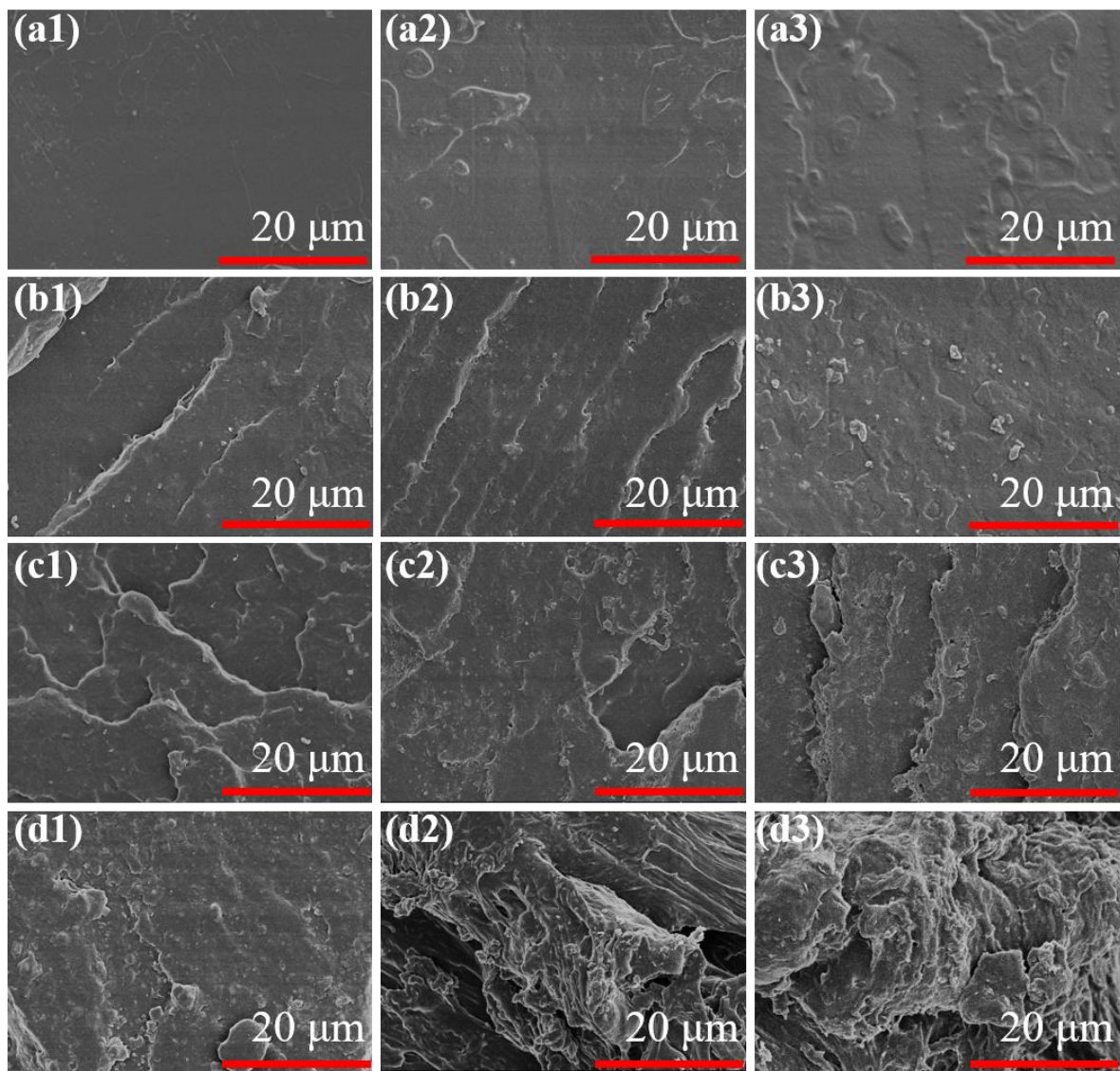

Fig. 9 SEM images of impact fractured surfaces for (a1-a3) Neat PLA, (b1-b2) 1K/10\%/1.1, (c1-c2) 1 K/20\%/1.1, (d1-d3)1K/30\%/1.1

prevented the crazing from further developing into cracks and greatly improved the toughness. As the CPU content increased, the surfaces became rough, accompanied by distinct plastic deformation and stress whitening.

\section{Conclusions}

In this paper, the PLA/CPU blends were successfully prepared by dynamic vulcanization and exhibited great mechanical properties. Simultaneously, we focused on the influence of PEG molecular weight, CPU content, and the ratio of -NCO/$\mathrm{OH}$ on the mechanical properties, crystallization properties, and compatibility of the blends. Through FTIR spectroscopy, gel content test, and TGA, sufficient evidence demonstrated that CPU and PLA-g-CPU were generated during the reaction process. According to the phase morphology analysis, thanks to PLA-g-CPU, there was phase separation in the blends, which reflected good compatibility between PLA and CPU. The impact fracture surfaces were analyzed, to reveal the fracture mechanism dominated by shear yield. Comparing the mechanical properties of different PLA/CPU blends, what we found was that the higher the molecular weight of PEG, the more unreacted PEG in the blends, which would cause PEG to precipitate on the surface and affect the use of materials. The CPU can effectively absorb external energy, whose content was directly related to the toughness. Most surprisingly, the impact strength by adding 30\% PEG into the blends was up to $72.05 \mathrm{~kJ} / \mathrm{m}^{2}$, which is much higher than that with only $20 \%$
PEG. No matter the higher or lower degree of cross-linking, the material properties would not be maximized. Finally, a conclusion was that fixing the ratio of $-\mathrm{NCO} /-\mathrm{OH}$ at 1.1 was the best choice in our study.

Dynamic vulcanization to enable the in-situ formation of CPU in the PLA matrix, overcoming the drawbacks of poor toughness of PLA, is thus proposed as a promising route for potentially widening its application range.

\section{Acknowledgments}

This work was supported by the National Natural Science Foundation of China (No. 51903092 and 52073107), the National Key Technology R\&D Program of China ((Grant Nos. 2020YFB1709301, 2020YFB1709304 and 2021YFC2101705).

\section{Conflict of interest}

There are no conflicts to declare.

\section{Supporting information}

Applicable.

\section{References}

[1] K. Hamad, M. Kaseem, M. Ayyoob, J. Joo, F. Deri, Progress in Polymer Science, 2018, 85, 83-127, doi: 10.1016/j.progpolymsci.2018.07.001.

[2] J. Chen, C. Rong, T. Lin, Y. Chen, J. Wu, J. You, H. Wang, 
Y. Li, Macromolecules, 2021, 10.1021/acs.macromol.0c02861.

[3] J. Huang, W. Zou, Y. Luo, Q. Wu, X. Lu, J. Qu, ES Energy and Environment, 2021, 12, 86-94, doi: 10.30919/esee8c1017. [4] J. Shojaeiarani, D. S. Bajwa, N. M. Stark, Carbohydrate Polymers, 2018, 190, 139-147, doi: 10.1016/j.carbpol.2018.02.069.

[5] X. Li, S. Gong, L. Yang, X. Xia, C. Linghu, J. Wang, Z. Luo, Polymer, 2021, 228, 123927, doi: 10.1016/j.polymer.2021.123927.

[6] C. Engineer, J. Parikh, A. Raval, Chemical Engineering Research and Design, 2011, 89, 328-334, doi: 10.1016/j.cherd.2010.06.013.

[7] O. A. Alimi, N. Bingwa, R. Meijboom, Homemade, Chemical Engineering Research and Design, 2019, 150, 116129, doi: 10.1016/j.cherd.2019.07.024.

[8] M. L. Robertson, J. M. Paxton, M. A. Hillmyer, ACS Applied Materials \& Interfaces, 2011, 3, 3402-3410, doi: 10.1021/am2006367.

[9] X. Zhang, B. Geng, H. Chen, Y. Chen, Y. Wang, L. Zhang, H. Liu, H. Yang, J. Chen, Chemical Engineering Journal, 2018, 334, 2014-2020, doi: 10.1016/j.cej.2017.11.102.

[10] J. C. C. Yeo, J. K. Muiruri, J. J. Koh, W. Thitsartarn, X. Zhang, J. Kong, T. T. Lin, Z. Li, C. He, Advanced Functional Materials, 2020, 30, 2001565, doi: 10.1002/adfm.202001565. [11] K. A. Athanasiou, G. G. Niederauer, C. M. Agrawal, Biomaterials, 1996, 17, 93-102, doi: 10.1016/01429612(96)85754-1.

[12] M. Puthumana, P. Santhana Gopala Krishnan, S. K. Nayak, International Journal of Polymer Analysis and Characterization, 2020, 25, 634-648, doi: 10.1080/1023666x.2020.1830650.

[13] T. Qiang, D. Yu, H. Gao, Journal of Applied Polymer Science, 2012, 124, 1831-1839, doi:10.1002/app.35224.

[14] K. Chang, M. L. Robertson, M. A. Hillmyer, ACS Applied Materials \& Interfaces, 2009, 1, 2390-2399, doi: 10.1021/am900514v.

[15] D. Garcia-Garcia, A. Carbonell-Verdu, M. P. Arrieta, J. López-Martínez, M. D. Samper, Polymer Degradation and Stability, 2020, 179, 109259, doi: 10.1016/j.polymdegradstab.2020.109259.

[16] M. Harada, T. Ohya, K. Iida, H. Hayashi, K. Hirano, H. Fukuda, Journal of Applied Polymer Science, 2007, 106, 1813-1820, doi: 10.1002/app.26717.

[17] D. Bai, H. Liu, H. Bai, Q. Zhang, Q. Fu, Macromolecules, 2017, 50, 7611-7619, doi: 10.1021/acs.macromol.7b01794.

[18] A. Gupta, V. Katiyar, ACS Sustainable Chemistry \& Engineering, 2017, 5, 6835-6844, doi: 10.1021/acssuschemeng.7b01059.

[19] S. Li, E. M. Woo, Polymer International, 2008, 57, 1242 1251, doi: 10.1002/pi.2469.

[20] Z. Y. Tan, X. F. Xu, S. L. Sun, C. Zhou, Y. H. Ao, H. X. Zhang, Y. Han, Polymer Engineering \& Science, 2006, 46, 1476-1484, doi: 10.1002/pen.20584.

[21] D. Bedő, B. Imre, A. Domján, P. Schön, G. J. Vancso, B.
Pukánszky, European Polymer Journal, 2017, 97, 409-417, doi: 10.1016/j.eurpolymj.2017.10.031.

[22] C. Koning, M. Van Duin, C. Pagnoulle, R. Jerome, Progress in Polymer Science, 1998, 23, 707-757, doi 10.1016/S0079-6700(97)00054-3.

[23] N. Zhang, X. Lu, Polymer Testing, 2016, 56, 354-363, doi: 10.1016/j.polymertesting.2016.11.003.

[24] N. Ning, S. Li, H. Wu, H. Tian, P. Yao, G.-H. Hu, M. Tian, L. Zhang, Progress in Polymer Science, 2018, 79, 61-97, doi: 10.1016/j.progpolymsci.2017.11.003.

[25] H. Liu, F. Chen, B. Liu, G. Estep, J. Zhang, Macromolecules, 2010, 43, 6058-6066, doi: $10.1021 / \mathrm{ma101108g.}$

[26] G. Jiang, J. Zhang, J. Ding, Y. Chen, Polymers for Advanced Technologies, 2021, 32, 2487-2498, doi: 10.1002/pat.5279.

[27] G. Wang, J. Zhao, L. H. Mark, G. Wang, K. Yu, C. Wang, C. B. Park, G. Zhao, Chemical Engineering Journal, 2017, 325, 632-646, doi: 10.1016/j.cej.2017.05.116.

[28] X. Xu, P. Fan, J. Ren, Y. Cheng, J. Ren, J. Zhao, R. Song, Composites Science and Technology, 2018, 168, 255-262, doi: 10.1016/j.compscitech.2018.10.003.

[29] A. S. de León, A. Domínguez-Calvo, S. I. Molina, Materials \& Design, 2019, 182, 108044, doi: 10.1016/j.matdes.2019.108044.

[30] H. Xiu, X. Qi, Z. Liu, Y. Zhou, H. Bai, Q. Zhang, Q. Fu, Composites Science and Technology, 2016, 127, 54-61, doi: 10.1016/j.compscitech.2016.02.025.

[31] W. Zhou, X. Chen, K. Yang, H. Fang, Z. Xu, Y. Ding, Applied Surface Science, 2022, 572, 151393, doi: 10.1016/j.apsusc.2021.151393.

[32] H.-Y. Mi, M. R. Salick, X. Jing, B. R. Jacques, W. C. Crone, X.-F. Peng, L.-S. Turng, Materials Science and Engineering: $\quad C, \quad 2013, \quad 33,4767-4776$, doi: 10.1016/j.msec.2013.07.037.

[33] G.-C. Liu, Y.-S. He, J.-B. Zeng, Q.-T. Li, Y.-Z. Wang, Biomacromolecules, 2014, 15, 4260-4271, doi: $10.1021 / \mathrm{bm} 5012739$.

[34] L. Liu, J. Hou, L. Wang, J. Zhang, Y. Duan, Industrial \& Engineering Chemistry Research, 2016, 55, 9907-9914, doi: 10.1021/acs.iecr.6b02122.

[35] P. Chanthot, N. Kaeophimmueang, P. Larpsuriyakul, C. Pattamaprom, Journal of Polymer Research, 2021, 28, 34, doi: 10.1007/s10965-020-02364-2.

[36] X. Lu, X. Wei, J. Huang, L. Yang, G. Zhang, G. He, M. Wang, J. Qu, Industrial \& Engineering Chemistry Research, 2014, 53, 17386-17393, doi: 10.1021/ie503092w.

[37] Z. Shakouri, H. Nazockdast, H. Sadeghi Ghari, Cellulose, 2020, 27, 215-231, doi: 10.1007/s10570-019-02775-5.

[38] M. S. Sánchez-Adsuar, E. Papon, J.-J. Villenave, Journal of Applied Polymer Science, 2001, 82, 3402-3408, doi: 10.1002/app.2201.

[39] Y.-S. He, J.-B. Zeng, G.-C. Liu, Q.-T. Li, Y.-Z. Wang, RSC Advances, 2014, 4, 12857-12866, doi: $10.1039 / \mathrm{c} 4 \mathrm{ra00718b}$. 
[40] S. Oprea, V.-O. Potolinca, V. Oprea, European Polymer Journal, 2016, 83, 161-172, doi: 10.1016/j.eurpolymj.2016.08.020.

[41] S. V. Karpov, V. P. Lodygina, V. V. Komratova, A. S. Dzhalmukhanova, G. V. Malkov, E. R. Badamshina, Kinetics and Catalysis, 2016, 57, 319-325, doi: 10.1134/s0023158416030058.

[42] B. Imre, D. Bedő, A. Domján, P. Schön, G. J. Vancso, B. Pukánszky, European Polymer Journal, 2013, 49, 3104-3113, doi: 10.1016/j.eurpolymj.2013.07.007.

[43] S. E. Fenni, F. Bertella, O. Monticelli, A. J. Müller, N. Hadadoui, D. Cavallo, ACS Omega, 2020, 5, 26421-26430, doi: 10.1021/acsomega.0c02765.

[44] X. Chen, J. A. Gardella Jr, T. Ho, K. J. Wynne, Macromolecules, 1995, 28, 1635-1642, doi: 10.1021/ma00109a041.

[45] K. Kojio, S. Nozaki, A. Takahara, S. Yamasaki, Journal of Polymer Research, 2020, 27, 140, doi: 10.1007/s10965020-02090-9.

[46] T. Gurunathan, J. S. Chung, S. K. Nayak, Journal of Polymers and the Environment, 2016, 24, 287-297, doi: 10.1007/s 10924-016-0771-x.

[47] Y. Lin, K.-Y. Zhang, Z.-M. Dong, L.-S. Dong, Y.-S. Li, Macromolecules, 2007, 40, 6257-6267, doi: 10.1021/ma070989a.

[48] M. Ma, K. Liu, H. Zheng, S. Chen, B. Wu, Y. Shi, X. Wang, Polymer International, 2018, 67, 1221-1228, doi: 10.1002/pi.5626.

[49] Y. He, Z. Yang, J. Qu, Composites Science and Technology, 2019, 169, 135-141, doi: 10.1016/j.compscitech.2018.11.002.

[50] A. M. Donald, E. J. Kramer, Journal of Materials Science, 1982, 17, 1871-1879, doi: 10.1007/bf00540402.

\section{Author Information}

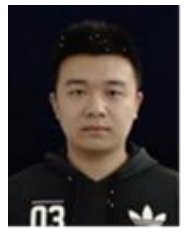

Mengjie Sheng is currently a M.S candidate in the group of Academician Qu Jinping, School of Chemistry and Chemical Engineering, Huazhong University of Science and Technology. His main research areas are functional phase change materials for energy storage, and processing and modification of polymer materials.

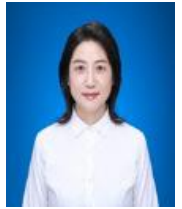

Lida $\boldsymbol{W u}$ is a engineer in food processing. She received her bachelor's degree from Jilin Agricultural University. Her main research area is modification of biodegradable materials.

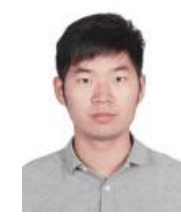

Xiaolong Li is currently a Ph.D. candidate in the group of Academician Qu Jinping, School of Chemistry and Chemical Engineering, Huazhong University of Science and Technology. He obtained the master degree from the College of
Materials and Metallurgy of Guizhou University. His main research areas are functional phase change materials for energy storage, and modification of biodegradable materials.

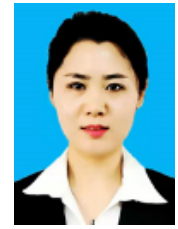

Han Yan, graduating from Beihua University, is mainly engaged in R\&D management. Her main research area is modification of biodegradable materials.

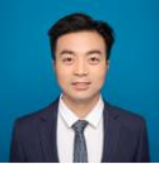

Xiang $\mathbf{L u}$ is currently an associate professor and Ph.D. supervisor in the Key Laboratory of Material Chemistry for Energy Conversion and Storage of Ministry of Education, School of Chemistry and Chemical Engineering at Huazhong University of Science and Technology, China. He received his Ph.D. from South China University of Technology. His research interests mainly focus on the design and fabrication of multi-functional materials especially for efficient energy utilization.

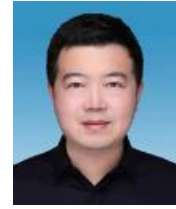

Yang $\boldsymbol{X u}$ is assistant to the General manager of Cofco (jilin) Bio-chemical Co. LTD. He holds a bachelor of Laws and master of Business Administration from Jilin University in China. His research interest in business administration.

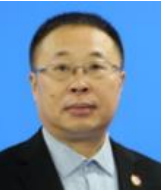

Yi Li, graduating from Jilin Institute of Technology, is a senior engineer, whose professional field is-modern and deep processing of corn.

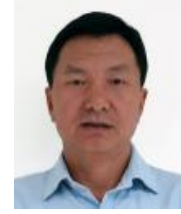

Yi Tong is a senior engineer in corn modern deep processing. He received his Ph.D. from Jilin University and majored in polymer chemistry and physics.

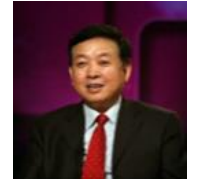

Jinping Qu is currently a professor in School of Chemistry and Chemical Engineering at Huazhong University of Science and Technology, and the academician of the Chinese Academy of Engineering. He received his Ph.D. from Sichuan University. His research interests mainly focus on design and fabrication of polymer processing equipment as well as the corresponding theory.

Publisher's Note: Engineered Science Publisher remains neutral with regard to jurisdictional claims in published maps and institutional affiliations. 